\title{
Geometry of nonabelian charged fluids
}

\author{
François Gay-Balmaz and Tudor S. Ratiu
}

Dedicated to the memory of our dear friend and mentor Jerry Marsden

\begin{abstract}
The goal of this paper is to derive the Hamiltonian structure of polarized and magnetized Euler-Maxwell fluids by reduction of the canonical symplectic form on phase space, and to generalize the dynamics to the nonabelian case. The Hamiltonian function we propose in this case, allows us to unify and relate in a simple way the main models of nonabelian charged fluids and their Hamiltonian structures.
\end{abstract}

\section{Contents}

1. Introduction 5

2. Hamiltonian reduction $\quad 7$

3. Dynamics of nonabelian charged fluids 11

$\begin{array}{ll}\text { References } & 18\end{array}$

\section{Introduction}

It is well-known that many conservative equations arising in hydrodynamics can be written in a Hamiltonian form, relative to noncanonical Poisson structures. A fundamental example consists of the adiabatic fluid and its magnetohydrodynamic (MHD) extension. Recall that this system consists of the motion equation for a fluid moving under the combined effects of pressure gradient and the net Lorentz force of the magnetic field created by the fluid in motion; the continuity equation for the mass density; advection of the specific entropy. In addition, the hypothesis of infinite conductivity leads to the conclusion that magnetic lines are frozen in the fluid, i.e., that they are transported along the particle paths.

2000 Mathematics Subject Classification. 37K65, 53C80, 76W05.

Key words and phrases. Reduction, Poisson brackets, nonabelian charged fluid, Yang-Mills field, Yang-Mills magnetohydrodynamics, Yang-Mills electrohydrodynamics, chromohydrodynamics.

The research of Tudor Ratiu is partially supported Swiss NSF grant 200020-126630. 
The noncanonical Hamiltonian structure for the adiabatic flow and its magnetohydrodynamic (MHD) extension was found by [20], [2], and [10]. One way to understand the occurrence of these noncanonical brackets is to obtain them from canonical ones. A first attempt in this direction is provided in [19] and [11], where the Poisson bracket is derived from Clebsch variables. A definitive answer is given in [17], [18], where it is shown that these brackets naturally arise by Poisson reduction of the canonical Poisson bracket in Lagrangian representation, the phase space of these systems being given by a semidirect product involving the diffeomorphism group $\operatorname{Diff}(\mathcal{D})$ of the fluid domain. For the incompressible Euler equations, this point of view is consistent with Arnold's treatment of perfect fluids [1]; see also [16].

Another important model in charged fluid dynamics is the Euler-Maxwell (EMF) fluid. This system consists of the motion equation for a charged fluid moving under the combined effects of pressure gradient and Lorentz forces; the continuity equation for the mass density; advection of the specific entropy; and Maxwell's equations for the electromagnetic fields $\mathbf{E}$ and $\mathbf{B}$ in the moving fluid medium, whose polarizability and magnetization are neglected. The Hamiltonian structure of this system was determined in [21], [22], and further studied in [11], [17]. The two Poisson structures obtained in these papers where shown in [4] to arise by reduction of the same canonical Poisson structure, at two different stages of the reduction process.

Poisson structures were also obtained for nonabelian fluid models such as YangMills magnetohydrodynamics (YM-MHD) and spin glass hydrodynamics (SGH) in [12], [13], for chromohydrodynamics (CHD) in [7], and complex fluids (CF) (such as liquid crystals) in [9]. These brackets were explained in [4] (for CHD), [5] and [6] (for YM-MHD, SGH, and CF) by reduction of the canonical structure on phase space. The basic idea is to consider as symmetry group the semidirect product $\operatorname{Diff}(\mathcal{D}) \subseteq \mathcal{F}(\mathcal{D}, \mathcal{O})$ of the diffeomorphism group with the gauge group, where $\mathcal{D}$ is the fluid domain and $\mathcal{O}$ is the structure group of the theory. For example, nematic liquid crystals, spin glass hydrodynamics or chromohydrodynamics involve the orthogonal group $S O(3)$ whereas superfluids such as ${ }^{4} \mathrm{He}$ involve $\mathcal{O}=U(1)$.

Consider now Euler-Maxwell dynamics in the more realistic case when the polarizability and magnetization of the moving fluid medium are not neglected. In this case, the Maxwell equations involve the four fields $\mathbf{E}, \mathbf{B}, \mathbf{H}, \mathbf{D}$, and the charged fluid moves under the effect of pressure gradient and moving-material effects, more complicated than the Lorentz force. The noncanonical Poisson structure of this system is determined in [8] and a nonabelian generalization was suggested in [13]. This bracket is not one of the abstract form described above, since it is neither an affine Lie-Poisson bracket (e.g. YM-MHD, SGH, and $\mathrm{CF}$ ) nor the direct product of a Lie-Poisson bracket and a canonical one (e.g. EMF or CHD). The goal of this paper is to derive this Poisson bracket by reduction of the canonical symplectic form on phase space and to generalize the dynamics to the nonabelian case. Moreover, the Hamiltonian function we propose in this case, allows us to unify the dynamics of charged fluids (in the standard and in the nonabelian case). Indeed, by particularizing this Hamiltonian, we recover MHD and electrohydrodynamics and their Hamiltonian structures, in the standard and in the nonabelian case. We also show that this Poisson bracket is isomorphic to that of CHD and how CHD is recovered by a using particularization of this same Hamiltonian. These results are 
summarized in Theorem 3.3 and could be useful for the Hamiltonian formulation of the electrodynamics of liquid crystal flows.

\section{Hamiltonian reduction}

The configuration space. The configuration space of the nonabelian charged fluid on a domain $\mathcal{D}$ in the presence of a self consistent Yang-Mills (YM) field with symmetry group $\mathcal{O}$ is

$$
Q=\left[\operatorname{Diff}(\mathcal{D})(\mathcal{F}(\mathcal{D}, \mathcal{O})] \times \Omega^{1}(\mathcal{D}, \mathfrak{o}) \ni(\eta, \chi, \bar{A}) .\right.
$$

Here $\operatorname{Diff}(\mathcal{D}) \subseteq \mathcal{F}(\mathcal{D}, \mathcal{O})$ denotes the semidirect product of the diffeomorphism group $\operatorname{Diff}(\mathcal{D})$ with the group of $\mathcal{O}$-valued functions $\mathcal{F}(\mathcal{D}, \mathcal{O})$; the group multiplication is

$$
\left(\eta_{1}, \chi_{1}\right)\left(\eta_{2}, \chi_{2}\right)=\left(\eta_{1} \circ \eta_{2},\left(\chi_{1} \circ \eta_{2}\right) \chi_{2}\right), \quad \eta_{1}, \eta_{2} \in \operatorname{Diff}(\mathcal{D}), \quad \chi_{1}, \chi_{2} \in \mathcal{F}(\mathcal{D}, \mathcal{O}),
$$

and $\Omega^{1}(\mathcal{D}, \mathfrak{o})$ denotes the space of $\mathfrak{o}$-valued one-forms on $\mathcal{D}$. The physical meaning of the variables is the following: the diffeomorphism $\eta: \mathcal{D} \rightarrow \mathcal{D}$ describes the Lagrangian motion of the fluid's particles, the gauge variable $\chi: \mathcal{D} \rightarrow \mathcal{O}$ describes internal degree of freedom in Lagrangian representation (nonabelian charge in the present case), and $\bar{A} \in \Omega^{1}(\mathcal{D}, \mathfrak{o})$ is the $Y M$ magnetic potential. The $Y M$ magnetic induction $\bar{B} \in \Omega^{2}(\mathcal{D}, \mathfrak{o})$ in Lagrangian representation is the curvature of $\bar{A}$ interpreted as a connection, namely:

$$
\bar{B}=\mathbf{d} \bar{A}+[\bar{A}, \bar{A}] .
$$

By definition, for any $\mu, \nu \in \Omega^{1}(\mathcal{D}, \mathfrak{o})$ the bracket $[\mu, \nu] \in \Omega^{2}(\mathcal{D}, \mathfrak{o})$ is defined by $[\mu, \nu](x)(u, v):=[\mu(x)(u), \nu(x)(v)]$ for any $x \in \mathcal{D}$ and $u, v \in T_{x} \mathcal{D}$.

To simplify the presentation, we will include the usual variables $\rho$ (mass density) and $S$ (entropy density) only later. The phase space of the charged fluid (ignoring $\rho$ and $S$ ) is thus

$$
T^{*} Q=T^{*}(\operatorname{Diff}(\mathcal{D}) \subseteq \mathcal{F}(\mathcal{D}, \mathcal{O})) \times T^{*} \Omega^{1}(\mathcal{D}, \mathfrak{o}) \ni\left(m_{\eta}, \kappa_{\chi}, \bar{A}, \overline{\mathbf{Y}}\right),
$$

where the variable $\bar{Y}$ canonically conjugated to $\bar{A}$ is related to the $Y M$ electric displacement field $\overline{\mathbf{D}}$ in Lagrangian representation by the relation $\overline{\mathbf{D}}=-\bar{Y}$. Here we have identified the cotangent bundle $T^{*} \Omega^{1}(\mathcal{D}, \mathfrak{o})$ with $\Omega^{1}(\mathcal{D}, \mathfrak{o}) \times \mathfrak{X}\left(\mathcal{D}, \mathfrak{o}^{*}\right)$ by using the $L^{2}$-paring associated to a fixed volume form on $\mathcal{D}$, where $\mathfrak{X}\left(\mathcal{D}, \mathfrak{o}^{*}\right)$ denotes the space of $\mathfrak{o}^{*}$-valued vector fields on $\mathcal{D}$, i.e., contravariant $\mathfrak{o}^{*}$-valued one-tensor fields.

The phase space is endowed with the canonical symplectic form on $T^{*} Q$ so that the equations of motion are the canonical Hamilton equations, symbolically written as

$$
\dot{q}=\frac{\partial H}{\partial q}, \quad \dot{p}=-\frac{\partial H}{\partial q},
$$

where $q$ corresponds to $(\eta, \chi, \bar{A})$ and $p$ corresponds to $\left(m_{\eta}, \kappa_{\chi}, \overline{\mathbf{Y}}\right)$.

Symmetry and Poisson reduction. Consider the natural action of the group $G=$ $\operatorname{Diff}(\mathcal{D}) \subseteq \mathcal{F}(\mathcal{D}, \mathcal{O}) \ni(\psi, \phi)$ on the configuration space given by right multiplication on the first two factors and gauge transformation of the connection, namely

$$
(\eta, \chi, \bar{A}) \cdot(\psi, \phi):=\left(\eta \circ \psi,(\chi \circ \psi) \phi, \operatorname{Ad}_{\phi^{-1}} \psi^{*} \bar{A}+\phi^{-1} T \phi\right) .
$$


If $H$ is a $G$-invariant Hamiltonian on $T^{*} Q$ relative to the cotangent lifted action, then it induces a reduced Hamiltonian on the quotient space $T^{*} Q / G$. We will identify the quotient space with

$$
\left(\mathfrak{X}(\mathcal{D})(S \mathcal{F}(\mathcal{D}, \mathfrak{o}))^{*} \times T^{*} \Omega^{1}(\mathcal{D}, \mathfrak{o}) \ni(m, \kappa, A, \mathbf{Y})\right.
$$

relative to the quotient map

$$
\left(m_{\eta}, \kappa_{\chi}, \bar{A}, \overline{\mathbf{Y}}\right) \mapsto(m, \kappa, A, \mathbf{Y}):=\left(m_{\eta}, \kappa_{\chi}, \bar{A}, \overline{\mathbf{Y}}\right) \cdot(\eta, \chi)^{-1}
$$

so that the reduced variables are

$$
\begin{array}{ll}
m=\left(m_{\eta} \circ \eta^{-1}\right) J \eta^{-1} \in \Omega^{1}(\mathcal{D}), & \kappa=\left(\kappa_{\chi} \chi^{-1} \circ \eta^{-1}\right) J \eta^{-1} \in \mathcal{F}\left(\mathcal{D}, \mathfrak{o}^{*}\right), \\
A=\psi_{*}\left(\operatorname{Ad}_{\phi} \bar{A}+\phi T \phi^{-1}\right) \in \Omega^{1}(\mathcal{D}, \mathfrak{o}), & \mathbf{Y}=\psi_{*}\left(\operatorname{Ad}_{\phi^{-1}}^{*} \overline{\mathbf{Y}}\right) \in \mathfrak{X}\left(\mathcal{D}, \mathfrak{o}^{*}\right),
\end{array}
$$

where $J \eta$ is the Jacobian determinant of the diffeomorphism $\eta$. This sort of reduction is sometimes called metamorphosis reduction since in the context of diffeomorphism group it was first developed for the metamorphosis approach to image dynamics ([14], [3]). Note that in the present case the Lie symmetry group is the semidirect product and not the diffeomorphism group only.

Note also that the symmetry group and the configuration space are the same as the ones used for the Euler-Yang-Mills (EYM) fluid in [4]. However, the action is not the same since, contrary to the EYM case, the group also acts on the YM fields.

In order to obtain the reduced Poisson structure, we shall use the following abstract reduction process (see $\S 10$ in [15]). Let $G$ be a Lie group acting on the right on a Poisson manifold $P$ and denote by $p \mapsto p g$ this action. Endow the space $T^{*} G \times P$ with the direct product Poisson structure. Consider the reduced space $\mathfrak{g}^{*} \times P$ with quotient map $\left(\alpha_{g}, p\right) \mapsto\left(\alpha_{g} g^{-1}, p g^{-1}\right)$. Then the reduced Poisson bracket $\{f, g\}_{\text {red }}$ on $\mathfrak{g}^{*} \times P$ is the sum of three terms

$$
\{f, g\}_{+}+\{f, g\}_{P}+\{f, g\}_{\text {coupling }}
$$

where the first term is the $(+)$ Lie-Poisson structure on $\mathfrak{g}^{*}$, the second term is the Poisson structure of $P$, and the coupling term is

$$
\{f, g\}_{\text {coupling }}=\left\langle\frac{\delta g}{\delta p},\left(\frac{\delta f}{\delta \mu}\right)_{P}\right\rangle-\left\langle\frac{\delta g}{\delta p},\left(\frac{\delta f}{\delta \mu}\right)_{P}\right\rangle
$$

where $\xi_{P} \in \mathfrak{X}(P)$, for $\xi \in \mathfrak{g}$, denotes the infinitesimal generator of the $G$-action on $P$. We now apply this result to our case by choosing the group $G=\operatorname{Diff}(\mathcal{D}) \subseteq \mathcal{F}(\mathcal{D}, \mathcal{O})$ and the Poisson manifold $P=T^{*} \Omega^{1}(\mathcal{D}, \mathfrak{o})$.

The reduced Poisson bracket has thus the form (2.2), where the Lie-Poisson structure is

$$
\{f, g\}_{+}(m, \kappa)=\left\langle m,\left[\frac{\delta f}{\delta m}, \frac{\delta g}{\delta m}\right]\right\rangle+\left\langle\kappa,\left[\frac{\delta f}{\delta \kappa}, \frac{\delta g}{\delta \kappa}\right]+\mathbf{d} \frac{\delta f}{\delta \kappa} \cdot \frac{\delta g}{\delta m}-\mathbf{d} \frac{\delta g}{\delta \kappa} \cdot \frac{\delta f}{\delta m}\right\rangle,
$$

and the Poisson structure on $P$ is the canonical bracket

$$
\{f, g\}_{c a n}(A, \mathbf{Y})=\left\langle\frac{\delta f}{\delta A}, \frac{\delta g}{\delta \mathbf{Y}}\right\rangle-\left\langle\frac{\delta g}{\delta A}, \frac{\delta f}{\delta \mathbf{Y}}\right\rangle .
$$

To obtain the coupling term, we use the formula of the infinitesimal generator

$$
(\mathbf{u}, \nu)_{T^{*} \Omega^{1}}(A, \mathbf{Y})=\left(-\operatorname{ad}_{\nu} A+£_{\mathbf{u}} A+\mathbf{d} \nu, \operatorname{ad}_{\nu}^{*} \mathbf{Y}+£_{\mathbf{u}} \mathbf{Y}+\mathbf{Y} \operatorname{div} \mathbf{u}\right)
$$


of the cotangent lifted action on $(A, \mathbf{Y})$ so that the coupling term is

$$
\begin{aligned}
\{f, g\}_{\text {coupling }}(m, \kappa, A, \mathbf{Y}) & \left\langle\left(\frac{\delta g}{\delta A}, \frac{\delta g}{\delta \mathbf{Y}}\right),\left(\frac{\delta f}{\delta m}, \frac{\delta f}{\delta \kappa}\right)_{T^{*} \Omega^{1}}(A, \mathbf{Y})\right\rangle \\
- & \left\langle\left(\frac{\delta f}{\delta A}, \frac{\delta f}{\delta \mathbf{Y}}\right),\left(\frac{\delta g}{\delta m}, \frac{\delta g}{\delta \kappa}\right)_{T^{*} \Omega^{1}}(A, \mathbf{Y})\right\rangle \\
= & \left\langle\frac{\delta g}{\delta A}, \mathbf{d}^{A} \frac{\delta f}{\delta \kappa}+£_{\frac{\delta f}{\delta m}} A\right\rangle+\left\langle\left[\frac{\delta f}{\delta \kappa}, \frac{\delta g}{\delta \mathbf{Y}}\right]-£_{\frac{\delta f}{\delta m}} \frac{\delta g}{\delta \mathbf{Y}}, \mathbf{Y}\right\rangle \\
& -\left\langle\frac{\delta f}{\delta A}, \mathbf{d}^{A} \frac{\delta g}{\delta \kappa}+£_{\frac{\delta g}{\delta m}} A\right\rangle-\left\langle\left[\frac{\delta g}{\delta \kappa}, \frac{\delta f}{\delta \mathbf{Y}}\right]-£_{\frac{\delta g}{\delta m}} \frac{\delta f}{\delta \mathbf{Y}}, \mathbf{Y}\right\rangle,
\end{aligned}
$$

where we used the notation $\mathbf{d}^{A} \nu:=\mathbf{d} \nu+[A, \nu]$ for the covariant differential of a function $\nu \in \mathcal{F}(\mathcal{D}, \mathfrak{o})$ relative to the connection $A$.

Adding the advected variables. As we said before, we need to add the usual advected quantities $\rho$ (mass density) and $S$ (entropy density) to the above variables. This means that the unreduced Hamiltonian $H$ is now defined on

$$
T^{*}\left[\operatorname{Diff}(\mathcal{D})(S \mathcal{F}(\mathcal{D}, \mathcal{O})] \times \mathcal{F}(\mathcal{D})^{2} \times T^{*} \Omega^{1}(\mathcal{D}, \mathfrak{o})\right.
$$

, where the space $\mathcal{F}(\mathcal{D})^{2} \ni(\rho, S)$ has been added. The Poisson structure is given by the sum of the canonical symplectic form on the cotangent bundles and the zero Poisson bracket on $\mathcal{F}(\mathcal{D})^{2}$. The symmetry group

$$
\operatorname{Diff}(\mathcal{D}) \subseteq \mathcal{F}(\mathcal{D}, \mathcal{O}) \ni(\psi, \phi)
$$

acts on $(\rho, S)$, only through its first factor, as $(\rho, S) \mapsto((\rho \circ \psi) J \psi,(\rho \circ \psi) J \psi)$. Note that $H$ is no longer on a cotangent bundle; however, using the tools of semidirect product reduction ([18]), we can obtain this space by reduction of a canonical cotangent bundle. When the variables $\rho$ and $S$ are included, the reduced space becomes

$$
(\mathfrak{X}(\mathcal{D}) \subseteq \mathcal{F}(\mathcal{D}, \mathfrak{o}))^{*} \times \mathcal{F}(\mathcal{D})^{2} \times T^{*} \Omega^{1}(\mathcal{D}, \mathfrak{o}) \ni(m, \kappa, \rho, S, A, \mathbf{Y})
$$

whose Poisson bracket is now the sum of (2.3), (2.4), and (2.5) plus the additional term

$$
\left\langle\rho, \mathbf{d} \frac{\delta f}{\delta \rho} \cdot \frac{\delta g}{\delta m}-\mathbf{d} \frac{\delta g}{\delta \rho} \cdot \frac{\delta f}{\delta m}\right\rangle+\left\langle S, \mathbf{d} \frac{\delta f}{\delta S} \cdot \frac{\delta g}{\delta m}-\mathbf{d} \frac{\delta g}{\delta S} \cdot \frac{\delta f}{\delta m}\right\rangle .
$$

Reduced Hamilton equations. We now give the reduced Hamilton equations $\dot{f}=\{f, h\}$ associated to the Poisson bracket obtained above and to a given reduced Hamiltonian $h$. These equations naturally involve the covariant divergence operator $\operatorname{div}^{A}: \mathfrak{X}\left(\mathcal{D}, \mathfrak{o}^{*}\right) \rightarrow \mathcal{F}\left(\mathcal{D}, \mathfrak{o}^{*}\right)$ defined as minus the $L^{2}$ adjoint of $\mathbf{d}^{A}$ and given by $\operatorname{div}^{A} \mathbf{Y}=\operatorname{div} \mathbf{Y}-\operatorname{Tr}\left(\operatorname{ad}_{A}^{*} \mathbf{Y}\right)$. The operators $\mathbf{d}^{A}$ and $\operatorname{div}^{A}$ extend naturally to $\mathfrak{o}$-valued $k$-forms and $\mathfrak{o}^{*}$-valued $k$-vector fields. For example, we will use below the formulas

$$
\mathbf{d}^{A} \mathbf{d}^{A} \nu=[B, \nu] \quad \text { and } \quad \operatorname{div}^{A} \operatorname{div}^{A} \mathbf{Y}=\operatorname{Tr}\left(\operatorname{ad}_{B}^{*} \mathbf{Y}\right)=\sum_{i<j} \operatorname{ad}_{B_{i j}}^{*} \mathbf{Y}^{i j},
$$

where $B:=\mathbf{d}^{A} A=\mathbf{d} A+[A, A]$ is the curvature two-form. 
A long but straightforward computation yields the reduced Hamilton equations

$$
\left\{\begin{array}{l}
\dot{m}+£_{\frac{\delta h}{\delta m}}^{d} m+\kappa \cdot \mathbf{d} \frac{\delta h}{\delta \kappa}+\rho \mathbf{d} \frac{\delta h}{\delta \rho}+S \mathbf{d} \frac{\delta h}{\delta S}+\mathbf{i}_{\frac{\delta h}{\delta A}} B \\
+\operatorname{div}^{A} \frac{\delta h}{\delta A} \cdot A-\mathbf{i}_{\mathbf{Y}} \mathbf{d} \frac{\delta h}{\delta \mathbf{Y}}-\operatorname{div} \mathbf{Y} \cdot \frac{\delta h}{\delta \mathbf{Y}}=0 \\
\dot{\kappa}+\operatorname{ad}_{\frac{\delta h}{\delta \kappa}}^{*} \kappa+\operatorname{div}\left(\kappa \frac{\delta h}{\delta m}\right)+\operatorname{div}^{A} \frac{\delta h}{\delta A}+\operatorname{Tr}\left(\operatorname{ad}_{\frac{\delta h}{\delta \mathbf{Y}}}^{*} \mathbf{Y}\right)=0 \\
\dot{\rho}+\operatorname{div}\left(\rho \frac{\delta h}{\delta m}\right)=0, \quad \dot{S}+\operatorname{div}\left(S \frac{\delta h}{\delta m}\right)=0 \\
\dot{A}+\mathbf{d}^{A} \frac{\delta h}{\delta \kappa}+£_{\frac{\delta h}{\delta m}} A=\frac{\delta h}{\delta \mathbf{Y}}, \quad \dot{\mathbf{Y}}+\operatorname{ad}_{\frac{\delta h}{\delta \kappa}}^{*} \mathbf{Y}+£_{\frac{\delta h}{\delta m}}^{d} \mathbf{Y}=-\frac{\delta h}{\delta A}
\end{array}\right.
$$

where we used the notation $£_{\mathbf{u}}^{d} \alpha=\nabla_{\mathbf{u}} \alpha+\nabla \mathbf{u}^{\top} \cdot \alpha+\alpha \operatorname{div} \mathbf{u}$ for the Lie derivative of a one-form thought of as a one-form density. In matrix notation and with respect to local coordinates these equations are equivalently written as

$$
\left[\begin{array}{c}
\dot{m}_{i} \\
\dot{\kappa}_{a} \\
\dot{\rho} \\
\dot{S} \\
\dot{A}_{i}^{a} \\
\dot{\mathbf{Y}}_{a}^{i}
\end{array}\right]=-\mathscr{B}\left[\begin{array}{c}
(\delta h / \delta m)^{k} \\
(\delta h / \delta \kappa)^{b} \\
\delta h / \delta \rho \\
\delta h / \delta S \\
(\delta h / \delta A)_{b}^{j} \\
(\delta h / \delta \mathbf{Y})_{j}^{b}
\end{array}\right]
$$

where the matrix $\mathscr{B}$ is given by

$$
\mathscr{B}=\left[\begin{array}{cccccc}
m_{k} \partial_{i}+\partial_{k} m_{i} & \kappa_{b} \partial_{i} & \rho \partial_{i} & S \partial_{i} & \partial_{j} A_{i}^{b}-A_{j, i}^{b} & \mathbf{Y}_{b}^{j} \partial_{i}-\partial_{k} \mathbf{Y}_{b}^{k} \delta_{i}^{j} \\
\partial_{k} \kappa_{a} & \kappa_{c} C_{b a}^{c} & 0 & 0 & \delta_{a}^{b} \partial_{j}-C_{c a}^{b} A_{j}^{c} & C_{b a}^{c} \mathbf{Y}_{c}^{j} \\
\partial_{k} \rho & 0 & 0 & 0 & 0 & 0 \\
\partial_{k} S & 0 & 0 & 0 & 0 & 0 \\
A_{k}^{a} \partial_{i}+A_{i, k}^{a} & \delta_{b}^{a} \partial_{i}+C_{c b}^{a} A_{i}^{c} & 0 & 0 & 0 & -\delta_{b}^{a} \delta_{i}^{j} \\
\partial_{k} \mathbf{Y}_{a}^{i}-\mathbf{Y}_{a}^{j} \delta_{k}^{i} \partial_{j} & C_{b a}^{c} \mathbf{Y}_{c}^{i} & 0 & 0 & \delta_{a}^{b} \delta_{j}^{i} & 0
\end{array}\right] .
$$

This recovers the noncanonical Poisson structure suggested in [13], matrix (2.33), for the dynamics of nonabelian charged fluids. The approach we present here justifies this choice since we show how it arises naturally by Poisson reduction of the canonical symplectic form on phase space.

This noncanonical Poisson structure is a natural generalization of the one of Yang-Mills MHD and spin glasses hydrodynamics discovered in [13], and obtained by affine Lie-Poisson reduction in [5], [6]. This structure is recovered from the present one by simply ignoring the variable $\mathbf{Y}$. We note that using the formula $\dot{B}=\mathbf{d}^{A} \dot{A}$, the equation for the curvature $B$ reads

$$
\dot{B}+\left[B, \frac{\delta h}{\delta \kappa}\right]+£_{\mathbf{v}} B=\mathbf{d}^{A} \frac{\delta h}{\delta \mathbf{Y}} .
$$




\section{Dynamics of nonabelian charged fluids}

Review of the Abelian case. In the abelian case, the Hamiltonian for the charged magnetized and polarized fluid is (see [8], eq. (11))

$$
h(m, \rho, S, A, \mathbf{Y})=\int_{\mathcal{D}}\left(-\frac{1}{2} \rho|\mathbf{v}|^{2}+m \cdot \mathbf{v}+\varepsilon(\rho, S, \mathbf{B}, \mathbf{D}, \mathbf{v})\right),
$$

where $\mathbf{D}:=-\mathbf{Y}$ is the electric displacement vector and $\mathbf{B}=\operatorname{curl} \mathbf{A}$, is the magnetic induction. As we will show later, to generalize this Hamiltonian to the nonabelian case, we first need to slightly modify it by adding a dependence on the abelian charge $\kappa$ and by replacing $m$ by $m-\kappa A$ in the expression of $h$. Consistently, in the abelian case the same equations emerge if we use the modified Hamiltonian, since the condition $\kappa=0$ is preserved by the dynamics and thus can be imposed at initial time. This is no longer true in the nonabelian case.

3.1. Nonabelian case. For general nonabelian fluids we thus choose the Hamiltonian

$$
h(m, \kappa, \rho, S, A, \mathbf{Y})=\int_{\mathcal{D}}\left(-\frac{1}{2} \rho|\mathbf{v}|^{2}+(m-\kappa A) \cdot \mathbf{v}+\varepsilon(\rho, S, B, \mathbf{D}, \mathbf{v}, \kappa)\right),
$$

where $\mathbf{v}=\mathbf{v}(m, \rho, S, A, \mathbf{Y})$ is the vector field defined by the implicit condition

$$
m-\kappa A-\rho \mathbf{v}^{b}+\frac{\partial \varepsilon}{\partial \mathbf{v}}(\rho, S, B, \mathbf{D}, \mathbf{v}, \kappa)=0 .
$$

Note that we have allowed a $\kappa$-dependence in the internal energy $\varepsilon$ since this does not introduce further difficulties. By the implicit function theorem, the above relation defines a unique vector field $\mathbf{v}$, provided the function $\varepsilon$ verifies the condition that

$$
u_{x} \mapsto \frac{\partial^{2} \varepsilon}{\partial \mathbf{v}^{2}}\left(r, s, B_{x}, D_{x}, v_{x}, k\right) \cdot u_{x}-r u_{x}
$$

is a bijective linear map in every fiber $T_{x} \mathcal{D}$.

We will use the following notations for the partial derivatives of the internal energy:

$$
\begin{gathered}
\mu:=\frac{\partial \varepsilon}{\partial \rho} \in \mathcal{F}(\mathcal{D}), \quad T:=\frac{\partial \varepsilon}{\partial S} \in \mathcal{F}(\mathcal{D}), \quad g:=-\frac{\partial \varepsilon}{\partial \mathbf{v}} \in \Omega^{1}(\mathcal{D}), \quad \zeta:=-\frac{\partial \varepsilon}{\partial \kappa} \in \mathcal{F}(\mathcal{D}, \mathfrak{o}), \\
E^{*}:=\frac{\partial \varepsilon}{\partial \mathbf{D}} \in \Omega^{1}(\mathcal{D}, \mathfrak{o}), \quad H^{*}:=\frac{\partial \varepsilon}{\partial B} \in \mathfrak{X}^{2}\left(\mathcal{D}, \mathfrak{o}^{*}\right),
\end{gathered}
$$

where the derivatives are understood as fiber derivatives. The physical interpretation is the following: $T$ is the temperature, $\mu$ is the chemical potential, $g$ is the electromagnetic momentum, $E^{*}$ represents the Yang-Mills electric field intensity, and $H^{*}$ represents the Yang-Mills magnetic field as measured in the fluid rest frame. The Yang-Mills electric field intensity $E \in \Omega^{1}(\mathcal{D}, \mathfrak{o})$ and the Yang-Mills magnetic field $H \in \mathfrak{X}^{2}\left(\mathcal{D}, \mathfrak{o}^{*}\right)$ as measured in the fluid rest frame are defined by the relations

$$
E^{*}=E-\mathbf{i}_{\mathbf{v}} B, \quad H^{*}=H+\mathbf{D} \wedge \mathbf{v} .
$$

Note that from (3.2), the total momentum is $m=\rho \mathbf{v}^{b}+\kappa A+g$.

If a Riemannian metric $g$ is fixed on $\mathcal{D}$, the derivative of the function $x \in \mathcal{D} \mapsto$ $\varepsilon(\rho(x), S(x), B(x), \mathbf{D}(x), \mathbf{v}(x)) \in \mathbb{R}$ is the one-form given by

$$
\mathbf{d}(\varepsilon(\rho, S, B, \mathbf{D}, \mathbf{v}))=\mu \mathbf{d} \rho+T \mathbf{d} S+E^{*} \cdot \nabla_{\mathbf{-}} \mathbf{D}+H^{*}: \nabla_{\mathbf{-}} B-g \cdot \nabla_{\mathbf{-}} \mathbf{v}+\zeta \mathbf{d} \kappa,
$$


where the symbol " : " means contraction on both indices. We have also assumed here that the horizontal partial derivative of $\varepsilon$ vanishes, i.e., relative to the chosen Riemannian metric on $\mathcal{D}$, the function $\varepsilon$ does not depend on the base point explicitly, as is the case in Euclidean space.

In order to obtain the motion equations for the nonabelian polarized and magnetized fluid, we compute the reduced equations (2.8) associated to the Hamiltonian (3.1). The functional derivatives are

$$
\begin{aligned}
& \frac{\delta h}{\delta m}=\mathbf{v}, \quad \frac{\delta h}{\delta \kappa}=\zeta-A \cdot \mathbf{v}, \quad \frac{\delta h}{\delta \rho}=-\frac{1}{2}|\mathbf{v}|^{2}+\mu, \quad \frac{\delta h}{\delta S}=\frac{\partial \varepsilon}{\partial S}=T, \\
& \frac{\delta h}{\delta A}=-\kappa \mathbf{v}-\operatorname{div}^{A} \frac{\partial \varepsilon}{\partial B}=-\kappa \mathbf{v}-\operatorname{div}^{A} H^{*}, \quad \frac{\delta h}{\delta \mathbf{Y}}=-\frac{\partial \varepsilon}{\partial \mathbf{D}}=-E^{*},
\end{aligned}
$$

so that $(2.8)$ reads

$$
\left\{\begin{array}{l}
\dot{m}+£_{\mathbf{v}}^{d} m+\kappa \cdot \mathbf{d}(\zeta-A \cdot \mathbf{v})+\rho \mathbf{d}\left(-1 / 2|\mathbf{v}|^{2}+\mu\right)+S \mathbf{d} T-\kappa \cdot \mathbf{i}_{\mathbf{v}} B \\
\quad-\mathbf{i}_{\operatorname{div}^{A} H^{*}} B-\operatorname{Tr}\left(\operatorname{ad}_{B}^{*} H^{*}\right) \cdot A-\operatorname{div}^{A}(\kappa \mathbf{v}) \cdot A-\mathbf{i}_{\mathbf{D}} \mathbf{d} E^{*}-\operatorname{div} \mathbf{D} \cdot E^{*}=0 \\
\dot{\kappa}+\operatorname{ad}_{\zeta-A \cdot \mathbf{v}}^{*} \kappa+\operatorname{div}(\kappa \mathbf{v})-\operatorname{div}^{A}(\kappa \mathbf{v})-\operatorname{Tr}\left(\operatorname{ad}_{B}^{*} H^{*}\right)+\operatorname{Tr}\left(\operatorname{ad}_{E^{*}}^{*} \mathbf{D}\right)=0 \\
\dot{\rho}+\operatorname{div}(\rho \mathbf{v})=0, \quad \dot{S}+\operatorname{div}(S \mathbf{v})=0 \\
\dot{A}+\mathbf{d}^{A}(\zeta-A \cdot \mathbf{v})+£_{\mathbf{v}} A=-E^{*}, \quad \dot{\mathbf{D}}+\operatorname{ad}_{\zeta-A \cdot \mathbf{v}}^{*} \mathbf{D}+£_{\mathbf{v}}^{d} \mathbf{D}=-\operatorname{div}^{A} H^{*}-\kappa \mathbf{v} .
\end{array}\right.
$$

We now rewrite the two last equations in terms of the fields $E$ and $H$ defined in (3.3). Using the formula $£_{\mathbf{v}} A=\mathbf{d}^{A}(A \cdot \mathbf{v})+\mathbf{i}_{\mathbf{v}} B$, the first equations reads $\dot{A}=-E-\mathrm{d}^{A} \zeta$ which yields

$$
\dot{B}=-\mathbf{d}^{A} E-[B, \zeta]
$$

For the second equation, we use the identity $\operatorname{div}^{A}(\mathbf{D} \wedge \mathbf{v})=\left(\operatorname{div}^{A} \mathbf{D}\right) \mathbf{v}-£_{\mathbf{v}}^{d} \mathbf{D}+$ $\operatorname{ad}_{A \cdot \mathbf{v}}^{*} \mathbf{D}$, to get

$$
\dot{\mathbf{D}}=-\operatorname{div}^{A} H-\left(\operatorname{div}^{A} \mathbf{D}+\kappa\right) \mathbf{v}+\operatorname{ad}_{\zeta}^{*} \mathbf{D}
$$

Note that the $\kappa$-equation simplifies to $\dot{\kappa}+\operatorname{ad}_{\zeta}^{*} \kappa-\operatorname{Tr}\left(\operatorname{ad}_{B}^{*} H^{*}\right)+\operatorname{Tr}\left(\operatorname{ad}_{E^{*}}^{*} \mathbf{D}\right)=0$. Using the formula $\operatorname{Tr}\left(\operatorname{ad}_{B}^{*} \mathbf{D} \wedge \mathbf{v}\right)=-\operatorname{Tr}\left(\operatorname{ad}_{\mathbf{i}_{\mathbf{v}} B}^{*} \mathbf{D}\right)$, this can be written in terms of $E$ and $H$ as

$$
\dot{\kappa}+\operatorname{ad}_{\zeta}^{*} \kappa-\operatorname{Tr}\left(\operatorname{ad}_{B}^{*} H\right)+\operatorname{Tr}\left(\operatorname{ad}_{E}^{*} \mathbf{D}\right)=0
$$

In order to rewrite the first equation of system (3.4) in a conservative form, we need to recall some facts concerning the divergence operator of tensor fields.

Divergence operator. Recall that the divergence of a vector field $X$ on a Riemannian manifold $(\mathcal{D}, g)$ is defined as the trace of the Levi-Civita covariant rerivative, that is, $\operatorname{div}(X):=\operatorname{Tr}(\nabla X)=\left\langle d x^{i}, \nabla_{\partial_{i}} X\right\rangle=(\nabla X)_{i}^{i}=\partial_{i} X^{i}+\Gamma_{i j}^{i} X^{j}$. Similarly, the divergence $\operatorname{div} S$ of a $(q, p)$ tensor field with $p \geq 1$ is the $(p-1, q)$ tensor field $\operatorname{div}(S)$ defined as the trace of the bilinear map $(\alpha, v) \mapsto \nabla_{v} S\left(\alpha,{ }_{-}\right)$so that in 
coordinates this reads

$$
\begin{aligned}
& \operatorname{div}(S)_{j_{1} \ldots j_{q}}^{i_{1} \ldots i_{p-1}}=(\nabla S)_{j_{1} \ldots j_{q} k}^{k i_{1} \ldots i_{p-1}} \\
& =\partial_{k} S_{j_{1} \ldots j_{q}}^{k i_{1} \ldots i_{p-1}}+\Gamma_{k l}^{k} S_{j_{1} \ldots j_{q}}^{l i_{1} \ldots i_{p-1}}+\Gamma_{k l}^{i_{1}} S_{j_{1} \ldots j_{q}}^{k l i_{2} \ldots i_{p-1}}+\text { all upper indices } \\
& \quad-\Gamma_{k j_{1}}^{l} S_{l j_{2} \ldots j_{q}}^{k i_{1} \ldots i_{p-1}}-\Gamma_{k j_{2}}^{l} S_{j_{1} l \ldots j_{q}}^{k i_{1} \ldots i_{p-1}}-\text { all lower indices. }
\end{aligned}
$$

We will need the following formulas, that are generalizations of the identity $\operatorname{div}(f X)=$ d $f \cdot X+f \operatorname{div}(X)$.

LEMma 3.1. Let $\nabla$ be a torsion free covariant derivative on $\mathcal{D}$.

(1) Given $X \in \mathfrak{X}\left(\mathcal{D}, \mathfrak{o}^{*}\right)$ and $\alpha \in \Omega^{1}(\mathcal{D}, \mathfrak{o})$, we have

$$
\begin{aligned}
\operatorname{div}(\alpha \otimes X) & =\nabla_{X} \alpha+\alpha \operatorname{div} X=\mathbf{i}_{X} \mathbf{d} \alpha+\alpha \operatorname{div} X+\nabla_{-} \alpha \cdot X \\
& =\mathbf{i}_{X} \mathbf{d} \alpha+\alpha \operatorname{div} X+\mathbf{d}(\alpha \cdot X)-\alpha \cdot \nabla_{-} X,
\end{aligned}
$$

where $(\alpha \otimes X)_{j}^{i}=\alpha_{j} X^{i}$.

(2) Given $B \in \Omega^{2}(\mathcal{D}, \mathfrak{o})$ and $b \in \mathfrak{X}^{2}\left(\mathcal{D}, \mathfrak{o}^{*}\right)$, we have

$$
\begin{aligned}
\operatorname{div}(B \cdot b) & =-\mathbf{i}_{\operatorname{div} b} B+b: \nabla_{-} B-b: \mathbf{i}_{-} \mathbf{d} B \\
& =-\mathbf{i}_{\operatorname{div}^{A} b} B-\left\langle A, \operatorname{Tr}\left(\operatorname{ad}_{B}^{*} b\right)\right\rangle+b: \nabla_{-} B-b: \mathbf{i}_{\mathbf{-}} \mathbf{d}^{A} B,
\end{aligned}
$$

where $(B \cdot b)_{j}^{i}=B_{j k} b^{i k}$ and $B: b=\sum_{i<j} B_{i j} b^{i j}$.

Proof. (1) The first identity follows easily from the definition. The second and third equalities follow, respectively, from the formulas $\mathbf{i}_{X} \mathbf{d} \alpha=\nabla_{X} \alpha-\nabla_{-} \alpha \cdot X$ and $\mathbf{d}(\alpha \cdot X)=\nabla_{-} \alpha \cdot X+\alpha \cdot \nabla_{-} X$.

(2) Using the definition of div and the properties of the covariant derivatives, we have

$$
\begin{aligned}
& \operatorname{div}(b \cdot B) \cdot \mathbf{u}=\operatorname{Tr}(\nabla(b \cdot B)) \cdot \mathbf{u}= \\
& \operatorname{Tr}(\nabla \operatorname{Tr}(b \otimes B)) \cdot \mathbf{u}=\nabla_{\partial_{i}} \operatorname{Tr}(b \otimes B)\left(d x^{i}, \mathbf{u}\right) \\
& =\operatorname{Tr}\left(\nabla_{\partial_{i}}(b \otimes B)\left(d x^{i}, \mathbf{u}\right)\right)=\operatorname{Tr}\left(\nabla_{\partial_{i}} b \otimes B\right)\left(d x^{i}, \mathbf{u}\right)+\operatorname{Tr}\left(b \otimes \nabla_{\partial_{i}} B\right)\left(d x^{i}, \mathbf{u}\right) \\
& =\nabla_{\partial_{i}} b\left(d x^{i}, d x^{k}\right) B\left(\mathbf{u}, \partial_{k}\right)+b\left(d x^{k}, d x^{i}\right) \nabla_{\partial_{i}} B\left(\partial_{k}, \mathbf{u}\right) \\
& =B(\mathbf{u}, \operatorname{div} b)+b: \nabla_{\mathbf{u}} B-b: \mathbf{i}_{\mathbf{u}} \mathbf{d} B
\end{aligned}
$$

To obtain the last two terms, we used the formula

$$
\mathbf{d} B(X, Y, Z)=\nabla_{X} B(Y, Z)+\nabla_{Y} B(Z, X)+\nabla_{Z} B(X, Y)
$$

to conclude that $b\left(d x^{k}, d x^{i}\right) \nabla_{\partial_{i}} B\left(\partial_{k}, \mathbf{u}\right)=b: \nabla_{\mathbf{u}} B-b: \mathbf{i}_{\mathbf{u}} \mathbf{d} B$.

The second identity in terms of the connection $A$ is obtained by using the formulas

$$
\begin{aligned}
\left(\operatorname{div}^{A} b\right)^{j} & =(\operatorname{div} b)^{j}-\operatorname{ad}_{A_{i}}^{*} b^{i j} \\
\mathbf{d}^{A} B(X, Y, X) & =\mathbf{d} B(X, Y, Z)+[A(X), B(Y, Z)] \\
& +[A(Y), B(Z, X)]+[A(Z), B(X, Z)],
\end{aligned}
$$

for $X, Y, Z \in \mathfrak{X}(\mathcal{D})$. 
Using this lemma, we can now rewrite the first equation of (2.8) in a conservative form. We have

$$
\begin{aligned}
& \kappa \cdot \mathbf{d}(\zeta-A \cdot \mathbf{v})-\kappa \cdot \mathbf{i}_{\mathbf{v}} B-\operatorname{div}^{A}(\kappa \mathbf{v}) \cdot A \\
& =\kappa \cdot \mathbf{d} \zeta-\kappa \cdot\left(\mathbf{d}^{A}(A \cdot \mathbf{v})+\mathbf{i}_{\mathbf{v}} B\right)-\operatorname{div}(\kappa \mathbf{v}) \cdot A \\
& =\kappa \cdot \mathbf{d} \zeta-\kappa \cdot £_{\mathbf{v}} A-\operatorname{div}(\kappa \mathbf{v}) \cdot A \\
& =\kappa \cdot \mathbf{d} \zeta-\operatorname{div}(\kappa A \otimes \mathbf{v})-\kappa A \cdot \nabla_{\mathbf{-}} \mathbf{v},
\end{aligned}
$$

so, using $m=\rho \mathbf{v}^{b}+\kappa A+g$, we can write

$$
\begin{aligned}
& £_{\mathbf{v}}^{d} m+\kappa \cdot \mathbf{d}(\zeta-A \cdot \mathbf{v})+\rho \mathbf{d}\left(-\frac{1}{2}|\mathbf{v}|^{2}+\mu\right)+S \mathbf{d} T-\kappa \cdot \mathbf{i}_{\mathbf{v}} B-\operatorname{div}^{A}(\kappa \mathbf{v}) \cdot A \\
& \quad=\kappa \cdot \mathbf{d} \zeta-\operatorname{div}(\kappa A \otimes \mathbf{v})-\kappa A \cdot \nabla_{\mathbf{-}} \mathbf{v}+\operatorname{div}(m \otimes \mathbf{v})+\left(m-\rho \mathbf{v}^{b}\right) \nabla_{\mathbf{-}} \mathbf{v}+\rho \mathbf{d} \mu+S \mathbf{d} T \\
& \quad=\mathbf{d}(\zeta \kappa+\rho \mu+s T)-\mu \mathbf{d} \rho-T \mathbf{d} S+g \nabla_{\mathbf{-}} \mathbf{v}+\operatorname{div}((m-\kappa A) \otimes \mathbf{v}) .
\end{aligned}
$$

Since $\mathbf{d}^{A} B=0$, we have $-\mathbf{i}_{\operatorname{div}^{A} H^{*}} B-\operatorname{Tr}\left(\operatorname{ad}_{B}^{*} H^{*}\right) \cdot A=\operatorname{div}\left(B \cdot H^{*}\right)-H^{*}: \nabla_{\_} B$ and $-\mathbf{i}_{\mathbf{D}} \mathbf{d} E^{*}-\operatorname{div} \mathbf{D} \cdot E^{*}=-\operatorname{div}\left(E^{*} \otimes \mathbf{D}\right)+\mathbf{d}\left(E^{*} \cdot \mathbf{D}\right)-E^{*} \cdot \nabla_{-} \mathbf{D}$, so that defining the $(1,1)$ tensor

$\mathbf{T}:=(m-\kappa A) \otimes \mathbf{v}+B \cdot H^{*}-E^{*} \otimes \mathbf{D}+q \delta, \quad$ with $\quad q:=\rho \mu+S T+E^{*} \cdot \mathbf{D}+\zeta \kappa-\varepsilon$, where $\delta$ is the $(1,1)$ tensor $\delta=\delta_{j}^{i} \frac{\partial}{\partial x^{i}} \otimes d x^{j}$, the first equation reads

$$
\dot{m}+\operatorname{div}(\mathbf{T})=0 .
$$

REMARK 3.2 (Three dimensional case). On a three dimensional Riemannian manifold $\mathcal{D}$, the Yang-Mills magnetic field $\mathbf{B} \in \mathfrak{X}(\mathcal{D}, \mathfrak{o})$ and the Yang-Mills induction field $\mathbf{H}^{*} \in \mathfrak{X}\left(\mathcal{D}, \mathfrak{o}^{*}\right)$ are defined by

$$
\mathbf{B}=(\star B)^{\sharp} \quad \text { and } \quad \mathbf{H}^{*}=\star H^{*},
$$

where $\star$ is the Hodge star operator acting on $\Omega^{k}(\mathcal{D}, \mathfrak{o})$ or, by duality, on $\mathfrak{X}^{k}\left(\mathcal{D}, \mathfrak{o}^{*}\right)$. Using the identity

$$
B \cdot H^{*}=\left(\mathbf{B}^{b} \cdot \mathbf{H}^{*}\right) \delta-\mathbf{B}^{b} \otimes \mathbf{H}^{*},
$$

the stress energy tensor $\mathbf{T}$ takes the familiar expression

$$
\mathbf{T}=(m-\kappa A) \otimes \mathbf{v}-\mathbf{B}^{b} \otimes \mathbf{H}^{*}-E^{*} \otimes \mathbf{D}+p \delta,
$$

with $p:=\rho \mu+S T+\zeta \kappa+\mathbf{B}^{b} \cdot \mathbf{H}^{*}+E^{*} \cdot \mathbf{D}-\varepsilon$

Returning to the general case and using (3.5), (3.6), (3.7), and (3.8), equations (3.4) can be written as

$$
\left\{\begin{array}{l}
\dot{m}+\operatorname{div}(\mathbf{T})=0 \\
\dot{\kappa}+\operatorname{ad}_{\zeta}^{*} \kappa-\operatorname{Tr}\left(\operatorname{ad}_{B}^{*} H\right)+\operatorname{Tr}\left(\operatorname{ad}_{E}^{*} \mathbf{D}\right)=0 \\
\dot{\rho}+\operatorname{div}(\rho \mathbf{v})=0, \quad \dot{S}+\operatorname{div}(S \mathbf{v})=0 \\
\dot{B}=-\mathbf{d}^{A} E-[B, \zeta] \\
\dot{\mathbf{D}}=-\operatorname{div}^{A} H-\left(\operatorname{div}^{A} \mathbf{D}+\kappa\right) \mathbf{v}+\operatorname{ad}_{\zeta}^{*} \mathbf{D}
\end{array}\right.
$$


Charge conservation. The total nonabelian charge is defined by

$$
Q:=\operatorname{div}^{A} \mathbf{D}+\kappa
$$

Using the formula $\operatorname{div}^{A}\left(\operatorname{ad}_{\nu}^{*} \mathbf{D}\right)=\operatorname{ad}_{\nu}^{*} \operatorname{div}^{A} \mathbf{D}+\operatorname{Tr}\left(\operatorname{ad}_{\mathbf{d}^{A} \nu}^{*} \mathbf{D}\right)$ and the $A$-equation $\dot{A}=-E-\mathbf{d}^{A} \zeta$, we compute the equation for $Q$. We have

$$
\begin{aligned}
\dot{Q}= & \operatorname{div}^{A} \dot{\mathbf{D}}-\operatorname{Tr}\left(\operatorname{ad}_{\dot{A}}^{*} \mathbf{D}\right)+\dot{\kappa} \\
= & -\operatorname{div}^{A} \operatorname{div}^{A} H-\operatorname{div}^{A}(Q \mathbf{v})-\operatorname{div}^{A} \operatorname{ad}_{\zeta}^{*} \mathbf{D} \\
& \quad+\operatorname{Tr}\left(\operatorname{ad}_{E}^{*} \mathbf{D}\right)+\operatorname{Tr}\left(\operatorname{ad}_{\mathbf{d}^{A} \zeta}^{*} \mathbf{D}\right)+\dot{\kappa} \\
= & -\operatorname{Tr}\left(\operatorname{ad}_{B}^{*} H\right)-\operatorname{div}^{A}(Q \mathbf{v})-\operatorname{ad}_{\zeta}^{*} \operatorname{div}^{A} \mathbf{D}+\operatorname{Tr}\left(\operatorname{ad}_{E}^{*} \mathbf{D}\right)+\dot{\kappa} \\
= & -\operatorname{div}^{A}(Q \mathbf{v})-\operatorname{ad}_{\zeta}^{*} \operatorname{div}^{A} \mathbf{D}-\operatorname{ad}_{\zeta}^{*} \kappa=-\operatorname{div}^{A}(Q \mathbf{v})-\operatorname{ad}_{\zeta}^{*} Q .
\end{aligned}
$$

We have thus obtained the equation of conservation of the charge

$$
\dot{Q}+\operatorname{div}^{A}(Q \mathbf{v})+\operatorname{ad}_{\zeta}^{*} Q=0, \quad \zeta=\frac{\partial \varepsilon}{\partial \kappa} .
$$

Consequently, the equations of motion can be equivalently written as

$$
\left\{\begin{array}{l}
\dot{m}+\operatorname{div}(\mathbf{T})=0 \\
\dot{Q}+\operatorname{div}^{A}(Q \mathbf{v})+\operatorname{ad}_{\zeta}^{*} Q=0 \\
\dot{\rho}+\operatorname{div}(\rho \mathbf{v})=0, \quad \dot{S}+\operatorname{div}(S \mathbf{v})=0 \\
\dot{B}=-\mathbf{d}^{A} E-[B, \zeta], \quad \dot{\mathbf{D}}=-\operatorname{div}^{A} H-Q \mathbf{v}+\operatorname{ad}_{\zeta}^{*} \mathbf{D} .
\end{array}\right.
$$

These are the equations for magnetized and polarized nonabelian charged fluids. In the physics literature, $\zeta=\frac{\partial \varepsilon}{\partial \kappa}$ is usually chosen to be zero.

Abelian case. We now consider the special case $\mathcal{O}=S^{1}$ and $\operatorname{dim}(\mathcal{D})=3$. Using the definitions of the fields $\mathbf{H}$ and $\mathbf{B}$ given in (3.9), we recover the equations

$$
\dot{\mathbf{B}}=-\operatorname{curl} \mathbf{E}, \quad \dot{\mathbf{D}}=\operatorname{curl} \mathbf{H}-q \mathbf{v}, \quad \operatorname{div} \mathbf{B}=0,
$$

where $q=\operatorname{div} \mathbf{D}+\kappa$. Note that the equation for $\kappa$ reads $\dot{\kappa}=0$, therefore, choosing $\kappa=0$ as initial condition, the stress energy tensor reads $\mathbf{T}=m \otimes \mathbf{v}-\mathbf{B}^{b} \otimes \mathbf{H}^{*}-$ $E^{*} \otimes \mathbf{D}+p \delta$ as in [8]. Therefore, in the abelian case, our equations consistently recover the equations for polarized and magnetized charged fluids (see equations (1a) $-(1 \mathrm{j})$ in $[8])$.

Particular case. Returning to the general nonabelian case, in the particular situation when $g=0$, that is, when $\varepsilon$ does not depend on the velocity $\mathbf{v}$, we have $\mathbf{v}^{b}=\frac{1}{\rho}(m-\kappa A)$ so that the Hamiltonian (3.1) becomes

$$
h(m, \kappa, \rho, S, A, \mathbf{Y})=\int_{\mathcal{D}} \frac{1}{2 \rho}|m-\kappa A|^{2}+\varepsilon(\rho, S, B, \mathbf{D}, \kappa) .
$$

As we will show in $\S 3.3$, the same Hamiltonian, but used with a different Poisson bracket, yields the EYM equation for chromohydrodynamics ([7], [4]).

3.2. Recovering YM-MHD \& YM-EHD. It was shown in [8] that, in the abelian case, ideal magnetohydrodynamics (MHD) and electrohydrodynamics (EHD) both emerge in Hamiltonian form as a regular limit of the charged polarized and magnetized fluid, by particularizing $\varepsilon$. Remarkably, despite the fact that our Hamiltonian slightly differs (even in the abelian case) this result still holds and generalizes to the nonabelian case. 
For YM-MHD, it suffices to choose

$$
\varepsilon(\rho, S, B, \mathbf{D}, \mathbf{v}, \kappa):=e(\rho, S)+\frac{1}{2}|B|^{2},
$$

in the Hamiltonian (3.1), where a bi-invariant inner product $\gamma$ is used to compute the norm of $B$. In this case, $H$ is given by $H_{b}^{i j}=H_{b}^{* i j}=g^{i n} g^{i m} \gamma_{a b} B_{m n}^{a}$, so that a computation similar to the above yields the motion equations

$$
\left\{\begin{array}{l}
\rho\left(\dot{\mathbf{v}}+\nabla_{\mathbf{v}} \mathbf{v}\right)^{b}=\mathbf{i}_{\operatorname{div}^{A} B+k \mathbf{v}} B-\mathbf{d} p, \quad p=\rho \mu+S T-e \\
\dot{\kappa}=0, \quad \dot{\rho}+\operatorname{div}(\rho \mathbf{v})=0, \quad \dot{S}+\operatorname{div}(S \mathbf{v})=0 \\
\dot{B}+£_{\mathbf{v}} B=0 .
\end{array}\right.
$$

These are the equations for YM-MHD, when the initial condition $\kappa=0$ is chosen.

For YM-EHD, choosing

$$
\varepsilon(\rho, S, B, \mathbf{D}, \mathbf{v}, \kappa):=e(\rho, S)+\frac{1}{2}|\mathbf{D}|^{2},
$$

yields $E_{i}^{b}=E_{i}^{* b}=g_{i j} \gamma^{a b} \mathbf{D}_{a}^{j}$, and we get the equations

$$
\left\{\begin{array}{l}
\rho\left(\dot{\mathbf{v}}+\nabla_{\mathbf{v}} \mathbf{v}\right)^{b}=\mathbf{i}_{\mathbf{D}} \mathbf{d} E+\operatorname{div}(\mathbf{D}) E-\mathbf{d} p, \quad p=\rho \mu+S T-e, \quad \mathbf{D}_{a}^{i}=\gamma_{a b} g^{i j} E_{j}^{b} \\
\dot{\kappa}+\operatorname{div}(\kappa \mathbf{v})=0, \quad \dot{\rho}+\operatorname{div}(\rho \mathbf{v})=0, \quad \dot{S}+\operatorname{div}(S \mathbf{v})=0 \\
\dot{\mathbf{D}}+£_{\mathbf{v}}^{d} \mathbf{D}=0 .
\end{array}\right.
$$

These are the equations for YM-EHD, when the initial condition $\kappa=0$ is chosen. Note that in the three dimensional case, the first and last equations recover the usual expressions

$$
\rho\left(\dot{\mathbf{v}}+\nabla_{\mathbf{v}} \mathbf{v}\right)=Q \mathbf{E}+\operatorname{curl} \mathbf{E} \times \mathbf{D}-\operatorname{grad} p \quad \text { and } \quad \dot{\mathbf{D}}=\operatorname{curl}(\mathbf{v} \times \mathbf{D})-Q \mathbf{v},
$$

where $Q=\operatorname{div} \mathbf{D} \in \mathcal{F}\left(\mathcal{D}, \mathfrak{o}^{*}\right)$ and $\mathbf{E} \in \mathfrak{X}(\mathcal{D}, \mathfrak{o})$ is defined by $\mathbf{E}^{i a}:=g^{i j} E_{j}^{a}$. The nonabelian charge $Q$ verifies the conservation law $\dot{Q}+\operatorname{div}(Q \mathbf{v})=0$.

3.3. Relation with EYM fluids. The Euler-Maxwell equations for charged fluids were extended to the nonabelian case in [7], where the corresponding Hamiltonian structure was also determined. These equations are known under the name of Euler-Yang-Mills (EYM) or chromohydrodynamics. This Hamiltonian structure was obtained by reduction of the canonical symplectic form in [4]. The reduction is associated to the same phase space as the one described in Section 2 but relative to a different symmetry, namely, the action (2.1) is replaced by the cotangent lifted action

$$
(\eta, \chi, A) \cdot(\psi, \phi):=(\eta \circ \psi,(\chi \circ \psi) \phi, A),
$$

where now $A$ is not acted on by the semidirect product. So the coupling term is now absent from the reduced Poisson structure, which consists simply of the sum of (2.3) and (2.4). In matrix representation, the Hamiltonian structure is therefore 
given by

$$
\mathscr{B}=\left[\begin{array}{cccccc}
m_{k} \partial_{i}+\partial_{k} m_{i} & \kappa_{b} \partial_{i} & \rho \partial_{i} & S \partial_{i} & 0 & 0 \\
\partial_{k} \kappa_{a} & \kappa_{c} C_{b a}^{c} & 0 & 0 & 0 & 0 \\
\partial_{k} \rho & 0 & 0 & 0 & 0 & 0 \\
\partial_{k} S & 0 & 0 & 0 & 0 & 0 \\
0 & 0 & 0 & 0 & 0 & -\delta_{b}^{a} \delta_{i}^{j} \\
0 & 0 & 0 & 0 & \delta_{a}^{b} \delta_{j}^{i} & 0
\end{array}\right]
$$

By applying a general result of [15] (see Proposition 10.3.2) to our case, there is a Poisson diffeomorphism that relates the Hamiltonian structures (2.10) and (3.17). It reads

$$
\begin{aligned}
(m, \kappa, A, \mathbf{Y}) \mapsto & ((m, \kappa)+\mathbf{J}(A, \mathbf{Y}), A, \mathbf{Y}) \\
& =\left(m-\mathbf{i}_{\mathbf{Y}} \mathbf{d} A-A \operatorname{div} \mathbf{Y}, \kappa-\operatorname{div}^{A} \mathbf{Y}, A, \mathbf{Y}\right)
\end{aligned}
$$

Remarkably, the EYM equations emerge with the same Hamiltonian $h$ as in (3.1), by choosing the particular case

$$
\varepsilon(\rho, S, B, E, \mathbf{v}, \kappa)=\frac{1}{2}|B|^{2}+\frac{1}{2}|E|^{2}
$$

(so $g=0$ ) and using the Hamiltonian structure (3.17) instead of (2.10). Indeed, in this case, the following equations are recovered

$$
\left\{\begin{array}{l}
\rho\left(\dot{\mathbf{v}}+\nabla_{\mathbf{v}} \mathbf{v}\right)^{b}=\left\langle\kappa, E-\mathbf{i}_{\mathbf{v}} B\right\rangle-\mathbf{d} p, \quad p=\rho \mu+S T-e, \\
\dot{\kappa}+\operatorname{div}^{A}(\kappa \mathbf{v})=0, \quad \dot{\rho}+\operatorname{div}(\rho \mathbf{v})=0, \quad \dot{S}+\operatorname{div}(S \mathbf{v})=0, \\
\dot{B}=-\mathbf{d}^{A} E, \quad \dot{E}=-\operatorname{div}^{A} B-\kappa \mathbf{v} .
\end{array}\right.
$$

As shown in [4], Gauss' law can be recovered by conservation of the momentum map associated to the cotangent lift of the gauge transformation $(\eta, \chi, A) \mapsto(\eta, \chi, A)$. $\psi=\left(\eta,\left(\psi^{-1} \circ \eta\right) \chi, \operatorname{Ad}_{\psi^{-1}} A+\psi^{-1} T \psi\right), \psi \in \mathcal{F}(\mathcal{D}, \mathcal{O})$ that commutes with (3.16). This momentum map is $\mathcal{J}_{\text {gau }}\left(m_{\eta}, \kappa_{\chi}, A, \mathbf{Y}\right)=-\left(\kappa_{\chi} \chi^{-1} \circ \eta^{-1}\right) J \eta^{-1}-\operatorname{div}^{A} \mathbf{Y}$ and thus induces the momentum map $\mathcal{J}_{\text {gau }}(m, \kappa, A, \mathbf{Y})=-\kappa-\operatorname{div}^{A} \mathbf{Y}$. Gauss' law

$$
\operatorname{div}^{A} E=\kappa
$$

is thus a consequence of Noether's theorem at zero momentum value.

Note that one can add the term $\frac{1}{2 \rho}|\kappa|^{2}$ to the Hamiltonian, where the norm is taken relative to a bi-invariant inner product $\gamma$ on $\mathfrak{o}$, and still obtain the correct equations (3.20). The resulting Hamiltonian

$$
h(m, \kappa, \rho, S, A, \mathbf{Y})=\int_{\mathcal{D}} \frac{1}{2 \rho}|m-\kappa \cdot A|^{2}+\frac{1}{2 \rho}|\kappa|^{2}+e(\rho, S)+\frac{1}{2}|E|^{2}+\frac{1}{2}|B|^{2},
$$

was considered in [4] and has the advantage to be nondegenerate and has thus an associated Lagrangian, the so called Kaluza-Klein Lagrangian

$$
\ell(\mathbf{v}, \nu, \rho, S, A, \mathbf{Y})=\int_{\mathcal{D}} \frac{\rho}{2}|\mathbf{v}|^{2}+\frac{\rho}{2}|A \cdot \mathbf{v}+\nu|^{2}-e(\rho, S)+\frac{1}{2}|E|^{2}-\frac{1}{2}|B|^{2} .
$$

We refer to [4] for the variational principle involved here as well as for the generalization to nontrivial principal bundles.

The results obtained in the paper are summarized in the following theorem. 
TheOREM 3.3 (Geometry of nonabelian fluids). Consider the configuration space $Q=\operatorname{Diff}(\mathcal{D}) \subseteq \mathcal{F}(\mathcal{D}, \mathcal{O}) \times \Omega^{1}(\mathcal{D}, \mathfrak{o})$ of the nonabelian charged fluid and its phase space $T^{*} Q$ endowed with the canonical symplectic form.

(1) Let the group $\operatorname{Diff}(\mathcal{D}) \subseteq \mathcal{F}(\mathcal{D}, \mathcal{O})$ act on $T^{*} Q$ by cotangent lift of the action (2.1). Then the associated reduced Poisson bracket yields the Hamiltonian structure (2.10) of the nonabelian charged magnetized and polarized fluid. The equations of motion are obtained by considering the Hamiltonian function

$$
h(m, \kappa, \rho, S, A, \mathbf{Y})=\int_{\mathcal{D}}-\frac{1}{2} \rho|\mathbf{v}|^{2}+(m-\kappa A) \cdot \mathbf{v}+\varepsilon(\rho, S, B, \mathbf{D}, \mathbf{v}, \kappa) .
$$

By specializing (see (3.12) and (3.14)) this Hamiltonian, two models of charged fluids (YM-MHD and YM-EHD) as well as their Hamiltonian structure are obtained.

(2) Let the group $\operatorname{Diff}(\mathcal{D}) \subseteq \mathcal{F}(\mathcal{D}, \mathcal{O})$ act on $T^{*} Q$ by cotangent lift of the action (3.16). Then the associated reduced Poisson bracket yields the Hamiltonian structure (3.17) of EYM fluids. The equations of motion are obtained by specialization (see (3.19)) of the same Hamiltonian $h$ as above.

The Hamiltonian structures (2.10) and (3.17) obtained in (1) and (2) are related by the Poisson diffeomorphism (3.18).

\section{References}

[1] Arnold, V. I. [1966], Sur la géométrie différentielle des groupes de Lie de dimenson infinie et ses applications à l'hydrodynamique des fluides parfaits, Ann. Inst. Fourier, Grenoble 16, 319-361.

[2] Dzyaloshinskii, I. E. and G. E. Volovick, [1980], Poisson brackets in condensed matter systems, Ann. Phys. 125, 67-97.

[3] Gay-Balmaz, F., D. D. Holm, and T. S. Ratiu [2010], Geometric dynamics of optimization, preprint.

[4] Gay-Balmaz, F. and T. S. Ratiu [2008a], Reduced Lagrangian and Hamiltonian formulation of Euler-Yang-Mills fluids, J. Sympl. Geom. 6(2), 189-237.

[5] Gay-Balmaz, F. and T. S. Ratiu [2008b] Affine Lie-Poisson reduction, Yang-Mills magnetohydrodynamics and superfluids, J. Phys. A: Math. Theor. 41, 344007, (24 pp)

[6] Gay-Balmaz, F. and T. S. Ratiu [2008c], The geometric structure of complex fluids, Adv. Appl. Math. 42 (2), 176-275.

[7] Gibbons, J., D. D. Holm and B. A. Kupershmidt [1983], The Hamiltonian structure of classical chromohydrodynamics, Physica D, 6, 179-194.

[8] Holm, D. D. [1986], Hamiltonian dynamics of a charged fluid, including electro- and magnetohydrodynamics, Physics Letters, 114A, 137-141.

[9] Holm, D. D. [2002], Euler-Poincaré dynamics of perfect complex fluids, in Geometry, Dynamics and Mechanics: 60th Birthday Volume for J.E. Marsden. P. Holmes, P. Newton, and A. Weinstein, eds., Springer-Verlag.

[10] Holm, D. D. and B. A. Kupershmidt [1983a], Poisson brackets and Clebsch representations for magnetohydrodynamics, multifluid plasmas, and elasticity, Physica 6D, 347-363.

[11] Holm, D. D. and B. A. Kupershmidt [1983b], Noncanonical Hamiltonian formulation of ideal magnetohydrodynamics, Physica $7 D, 330-333$.

[12] Holm, D. D. and B. A. Kupershmidt [1984], Yang-Mills magnetohydrodynamics: Nonrelativistic theory, Phys. Rev. D, 30, 2557-2560.

[13] Holm, D. D. and B. A. Kupershmidt [1988], The analogy between spin glasses and Yang-Mills fluids, J. Math. Phys. 29, 21-30.

[14] Holm D. D., A. Trouvé, and L. Younes [2009], The Euler-Poincaré theory of metamorphosis, Quart. Appl. Math 67, 661-685.

[15] Marsden, J. E., G. Misiołek, J.-P. Ortega, M. Perlmutter, and T. S. Ratiu [2007], Hamiltonian Reduction by Stages, Springer Lecture Notes in Mathematics, 1913, Springer-Verlag 2007.

[16] Marsden, J. E. and A. Weinstein [1983], Coadjoint orbits, vortices, and Clebsch variables for incompressible fluids, Phys. D 7, 305-323. 
[17] Marsden, J. E., A. Weinstein, T. S. Ratiu, R. Schmid and R. G. Spencer [1982], Hamiltonian systems with symmetry, coadjoint orbits and plasma physics, in Proc. IUTAM-IS1MM Symposium on Modern Developments in Analytical Mechanics (Torino 1982), 117, 289-340, Atti della Acad. della Sc. di Torino.

[18] Marsden, J. E., T. S. Ratiu and A. Weinstein [1984], Semidirect product and reduction in mechanics, Trans. Amer. Math. Soc., 281, 147-177.

[19] Morrison, P. J. [1982], Poisson Brackets for Fluids and Plasmas, in Mathematical Methods in Hydrodynamics and Integrability in Dynamical Systems. AIP Conf. Proc. 88, La Jolla, M. Tabor (Ed.)

[20] Morrison, P. J. and J. M. Greene [1980], Noncanonical Hamiltonian density formulation of hydrodynamics and ideal magnetohydrodynamics, Phys. Rev. Lett., 45, 790-794; errata 48 (1982), 569.

[21] Spencer, R. G. [1982], The Hamiltonian structure of multi-species fluid electrodynamics, in Mathematical Methods in Hydrodynamics and Integrability in Related Dynamical Systems. AIP Conf. Proc. 88, La Jolla, M. Tabor (Ed.).

[22] Spencer, R. G. and A. N. Kaufman [1982], Hamiltonian structure of two-fluid plasma dynamics, Phys. Rev. A 25, 2437-2439.

Laboratoire de Météorologie Dynamique, École Normale Supérieure/CNRS, Paris, FRANCE

E-mail address: gaybalma@lmd.ens.fr

Section de Mathématiques and Bernoulli Center, École Polytechnique Fédérale De Lausanne. CH-1015 Lausanne. Switzerland

E-mail address: tudor.ratiu@epfl.ch 